\title{
Long-Term Dietary Changes in Subjects with Glucose Galactose Malabsorption Secondary to Biallelic Mutations of SLC5A1
}

\author{
Alvin P. Chan ${ }^{1}$. Shweta S. Namjoshi ${ }^{2} \cdot$ Patricia M. Jardack $^{3} \cdot$ Lisa Maloney $^{4} \cdot$ Atrin Ardjmand $^{5}$. \\ Nicholas N. Jackson ${ }^{5}$ Martin G. Martin ${ }^{1}$
}

Received: 14 August 2020 / Accepted: 14 December 2020 / Published online: 12 January 2021

(c) The Author(s) 2021

\begin{abstract}
Background Glucose galactose malabsorption (GGM) is a congenital diarrheal disorder of intestinal Na+/glucose cotransport (SGLT1/SLC5A1). The required glucose and galactose-restricted diet has been well described in infancy, but long-term nutrition follow-up is limited.

Aim To perform a comprehensive nutritional assessment on a cohort of patients with GGM to gain insights into the consumption patterns within the population.

Methods A cross-sectional study examining dietary intake of a GGM cohort using prospective food records. The calories and nutrients of all foods, beverages, and condiments were analyzed with descriptive statistics and compared to intake patterns of age- and sex-matched NHANES groups.

Results The six patients were $0.7-26$ years old. Whole foods and vegetable fats were major parts of the diet, while dairy and added sweeteners were restricted. Compared to typical US intakes, mean macronutrient distribution was 88th percentile from fat, 18th percentile from carbohydrates, and 78th percentile from protein. Fructose consumption, as a proportion of total sugar intake, decreased with age, from 86.1 to 50.4\%. Meanwhile, glucose consumption increased with age, from 13.8 to $48.6 \%$ of sugar intake. However, the actual amount of glucose consumed remained low, equivalent to 4th percentile of US consumption level. Galactose intake was marginal throughout life.

Conclusions A GGM diet is a high-fat and high-protein/low-carbohydrate diet that is rich in fruits and vegetables but limited in dairy and added sugar. Relatively less fructose but more glucose is incorporated into the diet with age. Future studies should investigate the effects of the GGM diet on gut microbiome and long-term health.
\end{abstract}

Keywords Glucose galactose malabsorption $\cdot$ SLC5A1 - Sodium/glucose cotransporter $\cdot$ Nutrition $\cdot$ Diet $\cdot$ Low carbohydrate $\cdot$ High fat

\section{Abbreviations}

GGM Glucose galactose malabsorption

US United States

NHANES National Health and Nutrition Examination Survey

Supplementary Information The online version of this article (https://doi.org/10.1007/s10620-020-06792-4) contains supplementary material, which is available to authorized users.

Alvin P. Chan

alvinchan@mednet.ucla.edu

Extended author information available on the last page of the article

\section{Background}

Glucose galactose malabsorption (GGM, OMIM \#606824) is a rare autosomal recessive disorder of intestinal monosaccharide transport that results in lifelong, diet-induced diarrhea. Loss-of-function mutations of the sodium-glucose/galactose cotransporter (SGLT1/SLC5A1) result in the inability to transport the monosaccharides, glucose, and galactose, across the apical border of the intestinal epithelium, resulting in bloating, cramping, flatulence, and a dose-dependent osmotic diarrhea [1-3]. Following hydrolysis by specific enzymes, complex carbohydrates and disaccharides containing glucose and galactose, such as sucrose (glucose/fructose), lactose (glucose/galactose), and maltose (glucose/glucose), are also malabsorbed. The monosaccharide fructose is selectively transported across the apical 
membrane-facilitated transporter (GLUT5/SLC2A5), and its function is retained in GGM.

The standard of care for patients with GGM is lifelong restriction of dietary glucose and galactose. During infancy, the primary nutrition is a fructose-based formula, either as a Ross Carbohydrate-Free formula (Abbott Nutrition) with fructose supplementation, or a ready-made formula containing only fructose (Galactomin 19, Nutricia). Generally, by four to six months of age, low-carbohydrate foods, such as fruits and vegetables, can be introduced. Eventually, more carbohydrates may be incorporated, and some studies suggest improved tolerance to glucose and galactose with age [4-6]. The nutrition management of GGM during infancy has been well-described [6, 7], but long-term dietary followup is limited [5].

The aim of this study was to perform a meaningful dietary assessment on a cohort of patients with GGM to gain insights into consumption patterns within the population, while also drawing comparisons to the typical Western diet. This assessment not only forms a foundation for prospective nutritional studies in patients with GGM and other disorders of carbohydrate malabsorption, but also provides a practical resource for providers caring for patients with GGM.

\section{Materials and Methods}

\section{Study Procedures}

This cross-sectional, observational analysis of dietary intake of patients with GGM occurred between February and June of 2018. Given the rarity of GGM, patients of any age, gender, and race were recruited by convenience sampling via advertisements on social media networks supporting patients and families with GGM. The inclusion criterion was a genetically confirmed diagnosis of GGM. The exclusion criterion was diagnosis of any other disorders of malabsorption or carbohydrate metabolism due to confounding effects on dietary intake. All study materials and procedures were in accordance with and approved by the UCLA Institutional Review Board, \#17-001948.

Patients who expressed interest were screened for eligibility via a telephone encounter. Informed consent was obtained from all parents of patients and/or patients included in the study. Verbal and written instructions on study procedure were then provided, which included completion of a medical intake form and a written, prospective, three-day food record. To enhance the quality of the dietary data, the study dietitian reviewed the completed record with the patient/caregiver by telephone interview. A detailed medical history was also obtained by the study investigator over the telephone, including pertinent events leading up to the diagnosis of GGM, hospitalizations, medications, and current state of health (symptoms: bloating, constipation, diarrhea, abdominal pain, nausea, vomiting; bowel habits: Bristol stool type, number of stools per day). Past medical records, including hospital notes, clinic reports, laboratory studies, and/or growth charts, were provided by four patients. Medical documentation of a mutation in the SLC5Al gene confirming GGM was provided by all the patients.

\section{Study Population}

A sample of 15 patients with GGM living in seven countries responded to recruitment posts on social media networks. One patient was not eligible because the diagnosis of GGM was not confirmed by genetic testing. None of the patients had a known concurrent disorder that would influence appetite or result in digestive symptoms. Four chose not participate because of the anticipated demands of the three-day dietary collection. Of the 10 who originally agreed to participate, four were lost to follow up because of loss of interest and conflicting work and health obligations. Six English-speaking patients completed all study procedures.

\section{Dietary Analysis}

A detailed account of all foods, beverages, and condiments over a consecutive three-day period (Thursday, Friday, Saturday) was collected using a prospective food record. The types and amounts of food, calories, and nutrients were analyzed using the nutrition analysis software ESHA (Food Processor (C) 11.6.0, ESHA Research, Salem, OR), which is linked to a nutrient composition database (USDA SR-28). Food calorie totals were calculated in ESHA based on calories of the individual foods in a recipe or food list. Foods not found in the database or with missing values for any nutrients were entered manually by their respective ingredients. ESHA reports the content of fat, protein, alcohol, and water that are chemically analyzed in a laboratory, and ESHA measures the content of carbohydrate indirectly by subtracting the content of the above components from the total weight of the food sample ("carbohydrate by subtraction"). Therefore, the caloric contributions per grams of macronutrients do not precisely follow the commonly used 4-4-9 conversion factor from grams to calories for carbohydrate, protein, and fat, respectively.

\section{Statistical Methods}

The grams and calories of macronutrients (fat, carbohydrate, protein) and subtypes of macronutrients (fat: monounsaturated, polyunsaturated, saturated fat; carbohydrate: total sugar (including all naturally occurring and added sugar), fiber, starch; sugar: glucose, galactose, fructose, from monosaccharides and disaccharides) were examined 
using ESHA. Descriptive statistical analyses of all nutrients were performed for each patient and compared to reference values set by the US Dietary Reference Intakes and Dietary Guidelines. In order to compare dietary intakes between the GGM cohort and US groups, the consumption level for each nutrient was presented as a percentile relative to the typical intake of an age- and sex-matched US population using data from US National Health and Nutrition Examination Survey (NHANES III and NHANES 2018). Non-US patients were analyzed in this same way due to the lack of available worldwide nutrient database for sugar intake. Comparison of US groups was defined according to NHANES grouping: child 1-3 years old, male/female 4-8, male/female 9-13, male/female 14-18, and male/female 19-30. Patients with GGM less than 1 year old were analyzed in the "child 1-3 years old" group because of the lack of US nutrition monitoring data for infants. Tests for statistical significance were not performed due to small sample size. All data were analyzed using Stata SE version 15.1 (Stata Corp, LP; College Station, TX).

\section{Results}

\section{Patient Characteristics}

The six patients were from USA, Australia, Brazil, and Sweden. The youngest patient was a 0.7-year-old infant, and the oldest was a 25.8-year-old adult. The median age was 3.2 years. Four patients were females. All six patients presented with profound diarrhea at birth with a significant improvement after glucose and galactose elimination and were eventually diagnosed with GGM by molecular analysis of the SLC5Al gene.

All subjects were free of comorbid medical conditions, except one patient who had nephrolithiasis. Two patients were categorized as obese based on body mass index for age $>95$ th percentile, but they did not have documented features of metabolic syndrome, such as hypertension, insulin resistance, or dyslipidemia. All but the youngest patient reported regular symptoms, most commonly bloating and diarrhea. The oldest patient had highest reported frequency of symptoms. Table 1 summarizes clinical characteristics of the cohort of patients with GGM.

\section{Dietary Intake}

Whole foods (vegetables, fruit, meat, fish, and eggs) and vegetable fats (olive oil, vegetable oil) were key parts of the diet for all patients. Grains (bread, pasta, tortilla) comprised a small portion of the diet, while dairy products and sweetened foods were kept to a minimum. A fructose-based formula was the primary form of nutrition for the youngest patient (patient 1), but formula supplementation was also observed in the two 2-year-old toddler patients (patients 2 and 3). Foods and beverages with added sugar were occasionally found in the older patients' diet (patients 5 and 6). Supplementary Table 1 shows sample foods and beverages consumed by the patients. Energy intake was at the 54th percentile of the typical US population intake for the same sex and age (range: 27-86th percentile).

\section{Macronutrient Composition}

All of the patients consumed a diet that was high in fat and protein, but low in carbohydrate. The mean macronutrient distribution was $51 \%$ calories from fat (88th percentile for the typical US population consumption level for the same sex and age), 30\% from carbohydrates (18nd percentile), and 19\% from protein (78th percentile). Tables 2, 3, and 4 report the average daily intake of macronutrients and macronutrient subtypes in absolute amounts (grams), calories, and percentiles relative to the typical US population intake for the same sex and age.

\section{Carbohydrate}

Carbohydrates comprised the second highest source of calories. Carbohydrates consisted of complex carbohydrates (starch, fiber) and simple carbohydrates (sugar, including

Table 1 Clinical characteristics of cross-sectional cohort of patients with glucose galactose malabsorption

\begin{tabular}{|c|c|c|c|c|c|c|c|c|c|}
\hline ID & Race & Nationality & Sex & $\begin{array}{l}\text { Age at } \\
\text { present } \\
\text { (years) }\end{array}$ & Weight, kg & Height, $\mathrm{cm}$ & BMI, $\mathrm{kg} / \mathrm{m}^{2}$ (\%tile) & Symptoms & Bristol stool (\#/day) \\
\hline 1 & White & Brazilian & $\mathrm{F}$ & 0.7 & 8.9 & 71.5 & $17.5(65)$ & None & Type 4 (2) \\
\hline 2 & White & Swedish & M & 2.2 & 13.6 & 81.0 & 20.7 (99) & Bloating, diarrhea & Type 2, 4, 7 (1) \\
\hline 3 & White & USA & $\mathrm{F}$ & 2.4 & 15.2 & 87.6 & $19.8(99)$ & Bloating, constipation, diarrhea & Type 2, 6, 7 (2) \\
\hline 4 & Arab & Australian & M & 4.0 & 17.7 & 110.0 & $14.6(17)$ & Diarrhea, pain & Type 4, 7 (4) \\
\hline 5 & White & USA & $\mathrm{F}$ & 8.0 & 23.4 & 118.0 & $16.8(66)$ & Bloating & Type 4 (2) \\
\hline 6 & White & USA & $\mathrm{F}$ & 25.8 & 62.1 & 161.2 & $23.9(71)$ & $\begin{array}{l}\text { Bloating, constipation, diar- } \\
\text { rhea, pain, nausea }\end{array}$ & Type 1-7 (2) \\
\hline
\end{tabular}


monosaccharides and disaccharides) from fruits, vegetables, grains, dairy, and formula. Sugar was the predominant carbohydrate source for the youngest patient (patient 1), contributing $71 \%$ of total carbohydrate intake (36th percentile of the typical US population consumption level for the same sex and age). In comparison, sugar made up $48 \%$ of carbohydrates for the two toddler patients (29th percentile) (patients 2 and 3) and only 39\% for the older patients (11th percentile) (patients 4-6). Added sugar, which came from salad dressings, sauces, and sweetened foods and desserts, was minimal across all patients, contributing 0-1.6\% of total calories. Starch was also consumed in relatively small quantities (14th percentile). Fiber for all but the youngest patient was high (76th percentile for patients 2-6) (Tables 2 and 3).

\section{Fat}

For all patients, fats comprised the majority of calories. Fat consumption ranged between $1.7 \mathrm{~g} / \mathrm{kg}$ (patient 6) and $7.4 \mathrm{~g} /$ $\mathrm{kg}$ (patient 2) (Table 2). Fats came from polyunsaturated and monounsaturated fatty acids, such as peanuts and tree nuts (almond, pecan, cashew), fruit (avocado), oils (olive, vegetable), eggs, legumes (soy), and fish (salmon). Fats were also derived from saturated fatty acids, mainly from animal meat. Polyunsaturated, monounsaturated, and saturated fatty acids were consumed at $9 \%$ calories, $21 \%$ calories, and $15 \%$ calories, respectively (67th percentile, 93 th percentile, and 66th percentile of the typical US population consumption level for the same sex and age) (Table 3).

\section{Protein}

Protein provided the lowest caloric contribution to the diet. Protein intake ranged from $1.5 \mathrm{~g} / \mathrm{kg}$ (patient 6) to $7.3 \mathrm{~g} /$ $\mathrm{kg}$ (patient 2) (Table 2). Protein sources included meat, eggs, tree nuts, and fish. Extra protein was added into the diet using homemade protein-rich bread, soy flour, and protein shakes, for three of the patients (patients 2, 3, 5, respectively).

\section{Sugar Composition}

\section{Fructose}

Table 4 shows the amounts and percentages of total daily calories and sugar from fructose, glucose, and galactose. As a consequence of glucose and galactose restriction, fructose represented the largest percentage of sugar in the diet. The proportion of dietary fructose gradually decreased with age, from $86 \%$ of total sugar in the youngest patient (patient 1) to $50 \%$ in the oldest patient (patient 6). Compared to US intake levels, mean fructose consumption was at the 78th percentile for the younger patients 
(patients 1-3) and 17th percentile for the older patients (patients 4-6). Only for the second-oldest patient (patient 5) was the percentage of sugar from fructose actually lower than that from glucose, but the overall sugar intake for this patient was limited. The primary sources of fructose were formula (patient 1-3), fruits, and vegetables. Other less significant sources of fructose included added sugar (either sucrose or high fructose corn syrup) in sauces, dressings, yogurts, snacks, and desserts (patients 4-6).

\section{Glucose}

As the relative amount of fructose in the diet decreased, the amount of glucose intake increased with age, ranging from $13.8 \%$ of total sugar in the youngest patient (patient 1) to $48.6 \%$ in the oldest patient (patient 6). Despite this dietary shift toward more glucose, however, the mean glucose consumption remained low, comparable to the 4th percentile for contemporary US intake levels. Glucose came mainly from fruits, vegetables, and added sugar in condiments, snacks, and desserts, but also from dairy as lactose. Figure 1 shows

Table 3 Average daily intakes of macronutrients and macronutrient subtypes, as percentiles of typical US population consumption levels for the same sex and age, in cohort of patients with glucose galactose malabsorption

\begin{tabular}{rlllllllllllll}
\hline ID & Cal & Fat & Protein & Carb & Sat fat & Monofat & Polyfat & Sugar & Fiber & Starch & Fructose & Glucose & Galactose \\
\hline 1 & 27 & 72 & 24 & 16 & 48 & 94 & 35 & 36 & 42 & 7 & 85 & 1 & 5 \\
2 & 86 & 98 & 99 & 31 & 98 & 98 & 97 & 29 & 99 & 7 & 73 & 2 \\
3 & 80 & 97 & 85 & 44 & 46 & 87 & 90 & 28 & 86 & 47 & 76 & 1 \\
4 & 53 & 91 & 73 & 8 & 44 & 99 & 88 & 11 & 62 & 9 & 28 & 5 & 1 \\
5 & 27 & 77 & 91 & 2 & 85 & 86 & 35 & 1 & 46 & 7 & 4 & 1 \\
6 & 53 & 95 & 95 & 7 & 74 & 93 & 59 & 20 & 88 & 5 & 18 & 11 & 13 \\
\hline
\end{tabular}

Table 4 Amounts and percentages of daily calories and sugar from fructose, glucose, and galactose in cohort of patients with glucose galactose malabsorption

\begin{tabular}{|c|c|c|c|c|c|c|c|c|c|}
\hline \multirow[t]{2}{*}{ ID } & \multicolumn{3}{|l|}{ Total, g } & \multicolumn{3}{|c|}{$\%$ Calories } & \multicolumn{3}{|l|}{ \% Sugar } \\
\hline & Fructose & Glucose & Galactose & Fructose & Glucose & Galactose & Fructose & Glucose & Galactose \\
\hline 1 & 60.1 & 9.6 & 0.9 & 14.9 & 2.5 & 0.02 & 86.1 & 13.8 & 0.1 \\
\hline 2 & 47.4 & 17.4 & 0.1 & 11.7 & 4.6 & 0.02 & 73.0 & 26.8 & 0.2 \\
\hline 3 & 49.7 & 13.2 & 0.07 & 12.3 & 3.5 & 0.01 & 78.9 & 21.0 & 0.1 \\
\hline 4 & 33.1 & 28.1 & 0.2 & 6.9 & 6.4 & 0.05 & 53.9 & 45.8 & 0.3 \\
\hline 5 & 8.9 & 10.3 & 3.0 & 2.2 & 2.7 & 0.5 & 40.1 & 46.4 & 13.5 \\
\hline 6 & 24.9 & 24.0 & 0.5 & 6.2 & 6.2 & 0.08 & 50.4 & 48.6 & 1.0 \\
\hline
\end{tabular}

Fig. 1 Fructose and glucose as percentages of daily sugar intake in cohort of patients with glucose galactose malabsorption. Fructose intake (gray) was lower, while glucose intake (black) was higher, among older patients (each patient is represented by a different shape)

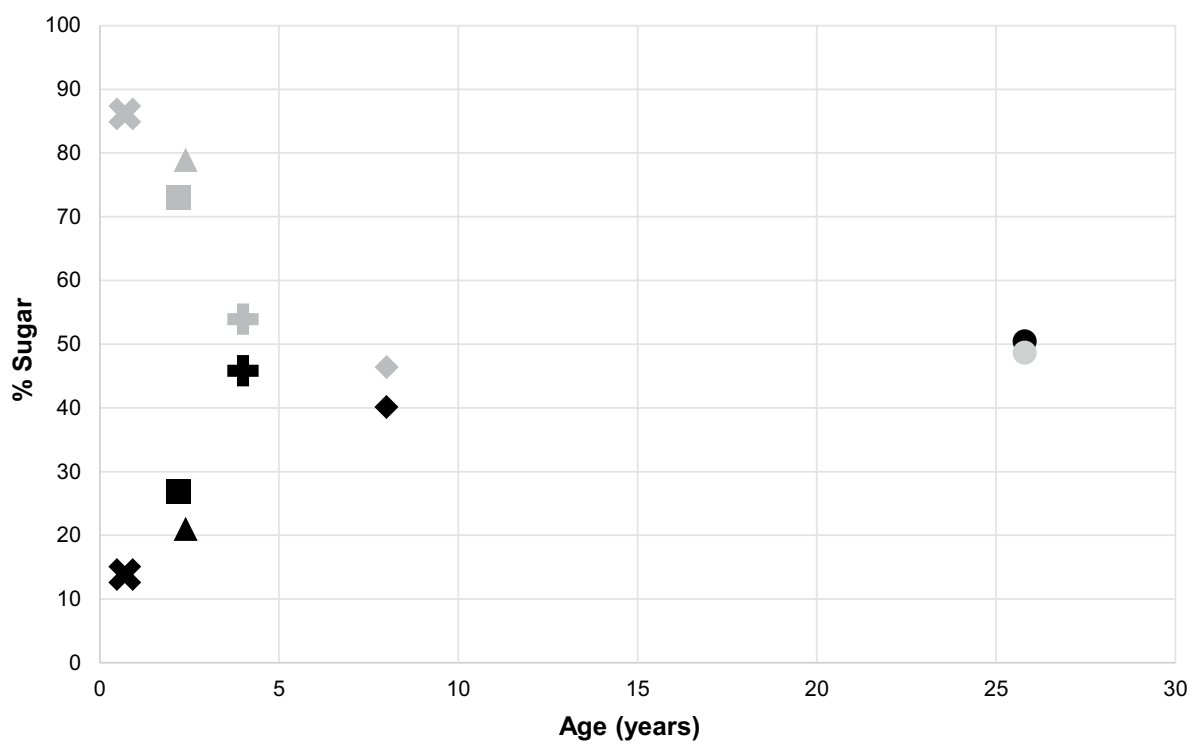


the inverse relationship between glucose and fructose as percentages of sugar in the diet with increasing age.

\section{Galactose}

Galactose intake was minimal for all patients, accounting for 0.1 to $13.5 \%$ all sugars consumed. Galactose consumption was at the 6th percentile for the typical US consumption level. Galactose was sourced almost exclusively from lactose in dairy from cow's milk and cheese. The cheeses consisted only the lower lactose varieties, such as mozzarella, cheddar, and Swiss. Except for the youngest patient (patient 1 ), dairy was consumed by all of the patients, with higher amounts consumed by the two oldest patients (patients 5 and 6). Besides dairy, galactose also came from avocados, although in much smaller amounts.

\section{Discussion}

To the best of our knowledge, this was the first detailed cross-sectional cohort analysis of dietary intake of infant, children, and adult patients with GGM. Our data suggest that a high-fat and high-protein/low-carbohydrate diet is followed by patients of all ages with GGM. This is consistent with a prior study of Amish children with GGM that also reported a high-fat and high-protein GGM diet [5]. Not surprisingly, fructose was the major source of sugar in the GGM diet because of the lifelong restriction to glucose and galactose. As shown, however, there was a shift in sugar intake toward more glucose and less fructose with increasing age-albeit at significantly lower levels when compared to age-matched national intake data. While the proportion of glucose in the diet was higher over time, the actual amount remained limited. Meanwhile, galactose intake was marginal throughout life.

In our GGM cohort, several trends were observed in the individual food group preferences as well as the macronutrient distribution. Within the fruits, there was a pattern of apples, pears, and mangos in the diet, all of which are high fructose containing fruit [8]. In the dairy group, there was a trend toward hard and aged cheeses, specifically cheddar and Swiss, because they are lower in lactose due to breakdown of lactose into lactic acid during the aging process. These trends reflect the natural affinity of the GGM group toward foods with less glucose and galactose to improve their gastrointestinal symptoms.

Total fat consumption within the GGM cohort was high compared to the US population. This was not surprising given the need to omit carbohydrates, specifically glucose and galactose, in GGM. The dietary fat included a mixture of specific types of fat, including polyunsaturated, monounsaturated, saturated, and trans fats. The polyunsaturated fat intake within our GGM cohort was 9\% calories, within the target $10 \%$ calories recommended by the National Heart, Lung, and Blood Institute (NHLBI) to reduce cardiovascular risk in pediatric patients, while the monosaturated fat intake was $21 \%$ calories, exceeding the target $10-15 \%$ calories [9-11].

Next are the saturated fats, found mostly in animal-based foods and even some plant foods, like nuts and palm oil. Mean saturated fat intake within our cohort was $15 \%$ calories, above the $8-10 \%$ recommended by the NHLBI and the Dietary Guidelines by Americans [9, 10]. It remains to be seen whether the health protective effects of high poly- and monounsaturated fat intake offset the potentially harmful consequences of the high saturated fat intake.

The final type of fat is trans fat, considered the most deleterious to cardiovascular health [12]. Trans fat intake in our cohort was minimal. Supplementary Table 2 displays the US daily nutritional goals and recommendations for various sex-age groups [9-11, 13].

Protein consumption was excessive in the GGM cohort, much greater than the amount necessary to meet the nutrient needs according to the Recommended Dietary Allowance [13] (Supplementary Table 2). Protein intake was also high relative to US consumption patterns because of the need for carbohydrate restriction in GGM. Protein is essential for growth and neurocognitive development, but it may also lead to glomerular hyperfiltration and accelerate kidney damage if the load exceeds kidney's excretory capabilities [14]. Although the kidneys are usually unaffected in GGM, impaired renal function associated with nephrolithiasis as a result of chronic dehydration has been documented in multiple case reports $[4,15]$. The single case of nephrolithiasis in our cohort (patient 4) was deemed by the medical team at the time to be more likely a result of dehydration than excessive protein consumption. Monitoring for complications associated with high-protein intake will be important for the long-term care of patients with GGM.

Carbohydrates were limited in the GGM cohort as a result of glucose and galactose restriction. Fructose was the primary sugar in the diet. Fruits and vegetables accounted for most of the fructose in the GGM cohort, while sugarsweetened beverages and grains, especially refined grains from processed foods, contributed the bulk of the fructose in the contemporary Western diet [16]. Added sugar intake among the GGM cohort was minimal at $<1 \%$ calorie intake, far below the recommended $<10 \%$ calorie intake by the Dietary Guidelines by Americans. Meanwhile, fiber intake was within the age-specific recommendations for most patients because of the high fruit and vegetable intake [13] (Supplementary Table 2).

Consistent with prior reports suggesting increased tolerance to glucose with time [4-6], our data showed an incremental decline in fructose consumption, alongside 
a tempered increase in glucose consumption, with age. Although the mechanisms underlying the age-dependent tolerance is unknown, one study noted that the administration of Lactobacillus acidophilus seemed to help to accelerate tolerance, suggesting possible intestinal flora adaptations over time [3]. Other studies speculate an increased colonic absorption from microbiota with age [17], genotype/phenotype variations in sugar tolerance [2], and presence of other glucose or galactose transport mechanisms besides SGLT1. GLUT2, once thought to be a glucose, galactose, and fructose transporter only on the basolateral membrane of the intestinal epithelium, however, had been shown in rat models to be an important apical transporter at very high luminal glucose concentrations [18], providing an alternative way to transport sugar across the brush border membrane. However, because the proposed mechanism by which GLUT2 inserts onto the apical membrane is contingent on SGLT1, it is unlikely to do so since the majority of the GGM variants in SGLT1 results in improper targeting to the apical membrane $[2,19]$. In another study in mice, data showing that SGLT1 null mice survived well on a glucose and galactose-free diet, but lost weight and eventually died after transitioning to a standard diet, further suggest that SGLT1 may be the primary apical transporter mediating glucose and galactose uptake [20]. Additionally, the presence or absence of GLUT2 did not seem to have any significant effect on glucose absorption in another study [21]. Based on our own experience, we hypothesize that the greater amounts of dietary glucose and galactose observed with older age may be the result of a higher functional reserve capacity of the gastrointestinal tract, smaller gastrointestinal caloric load (kcal/ $\mathrm{kg}$ ), and perhaps higher clinical tolerance to gastrointestinal symptoms (at the expense of a more liberalized diet). Collection of fecal output data in subsequent studies may help differentiate whether the increasing glucose consumption with advancing age is the result of poor dietary compliance or truly improving tolerance. Nonetheless, as our data demonstrated, the increasing amounts of glucose and galactose in the diet were only marginal.

\section{Limitations}

While the present study represents the most comprehensive dietary analysis of patients with GGM to date, the results should be interpreted in light of several limitations. The first limitation is the use of a self-reporting dietary assessment. Although a food record is validated instrument for the assessment of energy and nutrients, the method is prone to measurement error and bias. However, this study employed several strategies to reduce bias, including the use of prospective tracking over a three-day period and follow-up telephone interviews with a trained dietician to review the dietary details. The second limitation is the small sample size, an inherent challenge in the study of rare diseases. GGM is extremely rare, with approximately several hundred people diagnosed worldwide [22]. Like most other published studies on GGM which consist of single person to small group case reports, our study reported only six participants, limiting generalizability to this patient group as a whole. Our study, however, had the added advantage of including older children and an adult patient with GGM, which, to our knowledge, is the first-time patients beyond the toddler age has been evaluated from a nutritional standpoint. Because of the small sample size and experimental design, a final limitation is that this study cannot draw conclusions about the effects of diet on health outcomes within the GGM population. However, based on our dietary analysis, we suspect that patients with GGM, compared to their nonGGM counterparts, are less likely to suffer obesity-related metabolic disease because of their dietary choices, which we believe may be a more important predictor for health than the macronutrient and caloric content of the diet itself. Although micronutrient intakes were not evaluated in this study, a prior study showed that patients with GGM consume more vitamin $\mathrm{A}$, vitamin $\mathrm{D}$, iron, and zinc compared to those without GGM [5]. Future research requires a larger GGM cohort and longer-term follow-up to better understand how the individual foods and both the macro- and micronutrient components of the GGM diet impact health.

In conclusion, patients of all ages with GGM consume a high-fat and high-protein/low-carbohydrate diet that is rich in fruits and vegetables but limited in dairy and added sugar. Avoidance of dietary glucose and galactose is key, but increased intake of these sugars develops over time by an unclear mechanism that requires further investigation. Fructose, although consumed in higher quantities relative to glucose and galactose, makes up a smaller proportion of the GGM diet compared to the standard Western diet. More importantly, the fructose in the GGM diet comes primarily from fruits and vegetables, as opposed to sugarsweetened beverages and processed food in the Western diet. Future studies should investigate the effects of the GGM diet on intestinal transport mechanisms, gut microbiome, and long-term growth and health.

Author's contribution APC, SSN, and MGM designed research; APC, PMJ, and LM conducted research. APC, SSN, PMJ, AA, NNJ, LM, and MGM analyzed data. APC and SSN wrote the paper. APC, SSN, and MGM had primary responsibility for final content. All authors have read and approved the final manuscript.

Funding Research reported in this publication was supported by NIH National Center for Advancing Translational Science (NCATS) UCLA CTSI UL1TR001881 (A.P.C.), National Institute Of Diabetes And Digestive and Kidney Diseases of the National Institutes of Health under Award Number T32DK007180 (A.P.C.) and National Institute 
of Diabetes and Digestive and Kidney Disorders RC2 DK118640 (M.G.M.). The content is solely the responsibility of the authors and does not necessarily represent the official views of the National Institutes of Health.

\section{Compliance with Ethical Standards}

Conflict of interest None of the authors have potential financial, professional, or personal conflicts to disclose.

Ethical approval All procedures performed in studies involving human participants were in accordance with the ethical standards of the institutional and/or national research committee and with the 1964 Helsinki Declaration and its later amendments or comparable ethical standards.

Open Access This article is licensed under a Creative Commons Attribution-NonCommercial 4.0 International License, which permits any non-commercial use, sharing, adaptation, distribution and reproduction in any medium or format, as long as you give appropriate credit to the original author(s) and the source, provide a link to the Creative Commons licence, and indicate if changes were made. The images or other third party material in this article are included in the article's Creative Commons licence, unless indicated otherwise in a credit line to the material. If material is not included in the article's Creative Commons licence and your intended use is not permitted by statutory regulation or exceeds the permitted use, you will need to obtain permission directly from the copyright holder. To view a copy of this licence, visit http://creativecommons.org/licenses/by-nc/4.0/.

\section{References}

1. Turk E, Zabel B, Mundlos S, et al. Glucose/galactose malabsorption caused by a defect in the $\mathrm{Na}(+) /$ glucose cotransporter. Nature. 1991;350:354-356.

2. Martin MG, Turk E, Lostao MP, et al. Defects in Na+/glucose cotransporter (SGLT1) trafficking and function cause glucosegalactose malabsorption. Nat Genet. 1996;12:216-220.

3. Xin B, Wang H. Multiple sequence variations in SLC5A1 gene are associated with glucose-galactose malabsorption in a large cohort of Old Order Amish. Clin Genet. 2011;79:86-91.

4. Saadah OI, Alghamdi SA, Sindi HH, et al. Congenital glucosegalactose malabsorption: a descriptive study of clinical characteristics and outcome from Western Saudi Arabia. Arab J Gastroenterol. 2014;15:21-23.

5. Scanlan A, Hand R, Wolfram T, et al. Comparing dietary intake of amish children affected with glucose galactose malabsorption (GGM) to unaffected (UA) amish children. J Acad Nutr Diet. 2012;112:A35.

6. Abad-Sinden A, Borowitz S, Meyers R, et al. Nutrition management of congenital glucose-galactose malabsorption. J Am Diet Assoc. 1997;97:417-1421.

7. Ma M, Long Q, Fei C, et al. Nutrition management of congenital glucose-galactose malabsorption: case report of a Chinese infant. Medicine (Baltimore). 2019;98:e16828.
8. Matthews RH, Pehrsson PR, Farhat-Sabet M. Sugar content of selected foods: individual and total sugars. Washington, D.C.: U.S. Dept. of Agriculture, Human Nutrition Information Service; 1987.

9. Kleinman RE. Pediatric nutrition handbook. Elk Grove Village: American Academy of Pediatrics; 2009.

10. Expert Panel on Integrated Guidelines for Cardiovascular Health and Risk Reduction in Children and Adolescents. Expert panel on integrated guidelines for cardiovascular health and risk reduction in children and adolescents: summary report. Pediatrics 2011;128:S213-S256.

11. U.S. Department of Health and Human Services and U.S. Department of Agriculture. 2015-2020 Dietary Guidelines for Americans. 8th Edition. https://health.gov/our-work/food-and-nutri tion/2015-2020-dietary-guidelines/. Accessed March 23, 2020.

12. de Souza RJ, Mente A, Maroleanu A, et al. Intake of saturated and trans unsaturated fatty acids and risk of all cause mortality, cardiovascular disease, and type 2 diabetes: systematic review and meta-analysis of observational studies. BMJ. 2015;351:h3978.

13. National Research Council (US) Subcommittee. Recommended dietary allowances. 10th Edition. Washington, D.C.: National Academies Press (US); 1989.

14. Martin WF, Armstrong LE, Rodriguez NR. Dietary protein intake and renal function. Nutr Metab (Lond). 2005;2:25.

15. Ageel HI, Al-Agsam MA. Nephrolithiasis and nephrocalcinosis in children with glucose-galactose malabsorption: report of five cases. Am J Case Rep. 2017;5:190-195.

16. Vos MB, Kimmons JE, Gillespie C, et al. Dietary fructose consumption among US children and adults- the third national health and nutrition examination survey. Medscape J Med. 2008;10:160.

17. Phillips SF, McGill DB. Glucose-galactose malabsorption in an adult: perfusion studies of sugar, electrolyte, and water transport. Am J Dig Dis. 1973;18:1017-1024.

18. Kellett GL, Brot-Laroche E, Mace OJ, et al. Sugar absorption in the intestine: the role of GLUT2. Annu Rev Nutr. 2008;28:35-54.

19. Lam JT, Martin MG, Turk E, et al. Missense mutations in SGLT1 cause glucose-galactose malabsorption by trafficking defects. Biochim Biophys Acta. 1999;1453:297-303.

20. Gorboulev V, Schurmann A, Vallon V, et al. $\mathrm{Na}(+)$-D-glucose cotransporter SGLT1 is pivotal for intestinal glucose absorption and glucose-dependent incretin secretion. Diabetes. 2012;61:187-196.

21. Stümpel F, Burcelin R, Jungermann K, et al. Normal kinetics of intestinal glucose absorption in the absence of GLUT2: evidence for a transport pathway requiring glucose phosphorylation and transfer into the endoplasmic reticulum. Proc Natl Acad Sci USA. 2001;98:11330-11335.

22. Stenson PD, Ball EV, Mort M, et al. Human gene mutation database (HGMD): 2003 update. Hum Matat. 2003;21:577-581.

Publisher's Note Springer Nature remains neutral with regard to jurisdictional claims in published maps and institutional affiliations. 


\section{Affiliations}

\section{Alvin P. Chan ${ }^{1}$ (1) Shweta S. Namjoshi ${ }^{2}$. Patricia M. Jardack ${ }^{3} \cdot$ Lisa Maloney $^{4} \cdot$ Atrin Ardjmand $^{5}$. Nicholas N. Jackson ${ }^{5}$ Martin G. Martin ${ }^{1}$}

Shweta S. Namjoshi

namjoshi@stanford.edu

Patricia M. Jardack

pjardack@mednet.ucla.edu

Lisa Maloney

foodsolutionsolutions@outlook.com

Atrin Ardjmand

atrinardjmand@ucla.edu

Nicholas N. Jackson

njjackson@mednet.ucla.edu

Martin G. Martin

mmartin@mednet.ucla.edu

1 Division of Gastroenterology, Hepatology and Nutrition, Department of Pediatrics, Mattel Children's Hospital and David Geffen School of Medicine, University of California Los Angeles, Los Angeles, CA 90095, USA
2 Division of Gastroenterology, Hepatology and Nutrition, Department of Pediatrics, Lucile Packard Children's Hospital and Stanford University School of Medicine, Stanford University, Palo Alto, CA 94304, USA

3 Clinical Translational Research Center, Clinical and Translational Science Institute, David Geffen School of Medicine, University of California Los Angeles, Los Angeles, CA 90095, USA

4 Food Science Solutions, San Diego, CA 92101, USA

5 Department of Medicine Statistics Core, David Geffen School of Medicine, University of California Los Angeles, Los Angeles, CA 90095, USA 